\section{Über $\mathrm{NaBa}_{3} \mathrm{RuO}_{6}$ und $\mathrm{NaBa}_{3} \mathrm{IrO}_{6}$. Zwei Edelmetall-Oxometallate mit $\mathrm{Sr}_{4} \mathrm{PtO}_{6}$-Struktur}

On $\mathrm{NaBa}_{3} \mathrm{RuO}_{6}$ and $\mathrm{NaBa}_{3} \mathrm{IrO}_{6}$.

Two Noble Metal Oxometallates Showing $\mathrm{Sr}_{4} \mathrm{PtO}_{6}$ Structure

S. Frenzen, Hk. Müller-Buschbaum

Institut für Anorganische Chemie der ChristianAlbrechts-Universität, Olshausenstraße 40, D-24098 Kiel

Z. Naturforsch. 51b, 1204-1206 (1996); eingegangen am 26. Februar 1996

Sodium, Barium, Ruthenium, Iridium, Oxide, Crystal Structure

Single crystals of $\mathrm{NaBa}_{3} \mathrm{RuO}_{6}$ (I) and $\mathrm{NaBa}_{3} \mathrm{IrO}_{6}$ (II) have been prepared by crystallization from melts in closed silver tubes. The crystal structures were investigated by X-ray techniques. Both compounds crystallize with trigonal (rhombohedral) symmetry, space group $\mathrm{D}_{3 \mathrm{~d}^{6}}^{6} \mathrm{R} \overline{3} \mathrm{c}$, I: $a=10.107(2), c=11.872(2) \AA$, II: $a=10.128(2)$, $c=11.905(2) \AA, Z=6$. I and II are isostructural to the $\mathrm{Sr}_{4} \mathrm{PtO}_{6}$ type.

\section{Einleitung}

Die umfangreiche Literatur der Alkali- und Erdalkalimetall-Oxoruthenate und -Oxoiridate ist früher wiederholt zusammengestellt worden, so daß hier nur gemischte Alkali-ErdalkalimetallOxometallate der Edelmetalle zitiert werden. Aufzuführen sind $\mathrm{NaSr}_{4} \mathrm{Ru}_{2} \mathrm{O}_{9}$ und $\mathrm{KSr}_{4} \mathrm{Ru}_{2} \mathrm{O}_{9}$ [1], die unpubliziert blieben, da es seinerzeit nicht gelungen war, Einkristalle ausreichender Qualität darzustellen. Dennoch konnte erkannt werden, daß sie isotyp zu $\mathrm{NaBa}_{4} \mathrm{Cu}_{0.5} \mathrm{Pt}_{1.5} \mathrm{O}_{8}$ [2] sind. Die Kristallstrukturen dieser Stoffe zeigen Ketten, die aus flächenverknüpften $\mathrm{M}_{2} \mathrm{O}_{9}$-Oktaederdoppeln und trigonalen $\mathrm{NaO}_{6}$-Prismen gebildet werden. Wenig später wurde über $\mathrm{NaSr}_{3} \mathrm{RuO}_{6}$ [3] und $\mathrm{NaSr}_{3} \mathrm{IrO}_{6}[4]$ berichtet. Beide Verbindungen kristallisieren isotyp zu $\mathrm{Sr}_{4} \mathrm{PtO}_{6}$ und zeichnen sich durch Polyederstränge aus, die alternierend aus trigonalen $\mathrm{NaO}_{6}$-Prismen und $\mathrm{RuO}_{6^{-}}\left(\mathrm{IrO}_{6^{-}}\right) \mathrm{Ok}$ taedern aufgebaut sind. Es sei erwähnt, daß kürzlich auch eine silberhaltige Phase der Zusammensetzung $\mathrm{Ag}_{0.4} \mathrm{Na}_{2.3} \mathrm{Ca}_{4.3} \mathrm{RuO}_{8}$ [5] erhalten wurde,

\footnotetext{
* Sonderdruckanforderungen an Prof. Dr. Hk. MüllerBuschbaum.
}

die Verwandtschaft zur Natriumchloridstruktur aufweist. Es fällt auf, daß die Verbindungen des Bariums $\mathrm{Ba}_{4} \mathrm{LiRu}_{3} \mathrm{O}_{12}, \mathrm{Ba}_{4} \mathrm{NaRu}_{3} \mathrm{O}_{12}$ [6], $\mathrm{Ba}_{2} \mathrm{Na}_{0.5} \mathrm{Ru}_{0.5} \mathrm{TaO}_{6}$ [7], $\mathrm{BaM}_{0.5} \mathrm{Ir}_{0.5} \mathrm{O}_{2.75}$ und $\mathrm{BaM}_{0.25} \mathrm{Ir}_{0.75} \mathrm{O}_{3}(\mathrm{M}=\mathrm{Li}, \mathrm{Na})$ [8] nur an mikrokristallinem Material untersucht wurden. Die drei letztgenannten Stoffe gehören zu den kubischen Perowskiten, so daß die Alkali- und Edelmetallionen die Oktaederpositionen statistisch besetzen.

Soeben gelang erstmals die Darstellung von Einkristallen der Barium-Natrium-Oxometallate des Rutheniums und Iridiums, so daß zuverlässige Röntgenstrukturanalysen ausgeführt werden konnten.

\section{Experimentelles}

Zur Darstellung von Einkristallen der Verbindungen $\mathrm{NaBa}_{3} \mathrm{RuO}_{6}$ (I) und $\mathrm{NaBa}_{3} \mathrm{IrO}_{6}$ (II) wurden innige Gemenge von $\mathrm{Na}_{2} \mathrm{O}_{2}$ (Merck, p.a.), $\mathrm{BaO}$ (durch thermischen Abbau aus $\mathrm{Ba}(\mathrm{OH})_{2} \cdot 8 \mathrm{H}_{2} \mathrm{O}$ (Merck, p.a.) im Hochvakuum erhalten) und Ruthenium- bzw. Iridium-Pulver (beide Degussa, 99,9\%) im molaren Verhältnis 10:3:1 in geschlossenen Silberampullen auf $800^{\circ} \mathrm{C}$ erhitzt und die Temperatur $21 \mathrm{~d}$ gehalten. Anschließend wurde zunächst langsam mit $0,5{ }^{\circ} \mathrm{C} / \mathrm{h}$ auf $650^{\circ} \mathrm{C}$, dann rascher mit $20^{\circ} \mathrm{C} / \mathrm{h}$ auf $300^{\circ} \mathrm{C}$ abgekühlt. Der Überschuß an $\mathrm{Na}_{2} \mathrm{O}_{2}$ wirkt als Oxidationsmittel für Ruthenium und Iridium und zugleich als Schmelzmittel für die Bildung von Einkristallen. Nach dem Öffnen der Silberbömbchen unter trockener Gasatmosphäre wurden aus dem inhomogenen Reaktionsprodukt schwarze Einkristalle isoliert. Diese zeigten für I die Gestalt trigonaler Plättchen, für II waren es hexagonale Stäbchen. Die analytische Untersuchung erfolgte mit energiedispersiver Röntgenspektrometrie (Elektronenmikroskop Leitz SR 50, EDX-System Link AN 10000) unter Anwendung standardfreier Meßtechnik. Bei Normierung auf die Edelmetalle ergaben sich die Werte: I Na:Ba:Ru = 0,8:2,5:1 und II $\mathrm{Na}: \mathrm{Ba}: \operatorname{Ir}=1,1: 3,3: 1$.

Mit Weissenberg-Aufnahmen und Vierkreisdiffraktometermessungen wurden die in Tab. I wiedergegebenen kristallographischen Daten ermittelt. Mit dem Programm SHELXL-93 [9] wurden die von $\mathrm{NaSr}_{3} \mathrm{IrO}_{6}$ übernommenen Parameter verfeinert. Für Substanz II stellte sich heraus, daß es sich um einen Reverse/Obverse-Zwilling handelte. Durch die Überlagerung der beiden Individuen waren die Reflexe $h k l$ mit $l=3 n$ verfälscht. Wie auch schon für $\mathrm{NaSr}_{3} \mathrm{IrO}_{6}[4]$ beschrieben, wurden 
Tab. I. Kristallographische Daten und Messparameter für $\mathrm{NaBa}_{3} \mathrm{RuO}_{6}$ (I) und $\mathrm{NaBa}_{3} \mathrm{IrO}_{6}$ (II) mit Standardabweichungen in Klammern.

Kristallsystem

Raumgruppe

Gitterkonstanten $[\AA]$

Zellvolumen $\left[\AA^{3}\right]$

Anzahl Formeleinheiten

Diffraktometer

Strahlung/Monochromator

Röntg. Dichte (berechnet)

Absorptionskoeffizient

Extinktionskoeffizient

$\mathrm{F}(000)$

$2 \theta$-Bereich

Messmodus

Messzeit/Schritt

Korrekturen

Vermessene Reflexe

Symmetrieunabhängige Reflexe

Symmetrieunabhängige Reflexe

für eine Zwillingsspezies

Reflexe für $(\mathrm{I}>2 \sigma(\mathrm{I}))$

Restraints/Parameter

Goodness-of-fit für $\mathrm{F}^{2}$

Gütefaktoren anisotrop

(alle Reflexe)

\begin{tabular}{|c|c|}
\hline (I) & (II) \\
\hline $\begin{array}{l}\text { trigonal-rho } \\
D^{6}-R^{3} \mathrm{C}\end{array}$ & $\begin{array}{l}\text { mboedrisch } \\
\text { Nr. } 167\end{array}$ \\
\hline$=10,107(2)$ & $a=10,128(2)$ \\
\hline$=11,872(2)$ & $c=11.905(2)$ \\
\hline $1050.3(2)$ & $\begin{array}{l}1057.6(3) \\
6\end{array}$ \\
\hline Siemens & AED2 \\
\hline $\operatorname{MoK} \alpha /($ & jraphit \\
\hline $5,996 \mathrm{~g} / \mathrm{cm}^{3}$ & $6,813 \mathrm{~g} / \mathrm{cm}^{3}$ \\
\hline $18,785 \mathrm{~mm}^{-1}$ & $35,370 \mathrm{~mm}^{-1}$ \\
\hline $0,00169(7)$ & $0.00064(3)$ \\
\hline 1620 & 1818 \\
\hline $8,0-70,0^{\circ}$ & $8,2-69,90^{\circ}$ \\
\hline$\Omega / 2 \theta$ & $\Omega / 2 \theta$ \\
\hline $1-3 s$ & $2-5 \mathrm{~s}$ \\
\hline $\begin{array}{l}\text { Untergrund. } \\
\text { und Lore }\end{array}$ & $\begin{array}{l}\text { olarisations- } \\
\text { tzfaktor }\end{array}$ \\
\hline 1032 & 2082 \\
\hline 519 & 523 \\
\hline & 343 \\
\hline & 267 \\
\hline ) / 20 & $0 / 20$ \\
\hline 1,116 & 0,937 \\
\hline $\begin{array}{l}=0,027 \\
22=0,041\end{array}$ & $\begin{array}{l}R 1=0,041 \\
w R 2=0,039\end{array}$ \\
\hline
\end{tabular}

die Reflexe eines Zwillingsindividuums eliminiert. Mit dem zweiten Reflexdatensatz wurden die Parameter verfeinert. Die endgültigen Werte stellt Tab. II zusammen. In Tab. III sind die wichtigsten Metall-Sauerstoff-Abstände wiedergegeben*.

Tab. II. Lageparameter und isotrope Temperaturfaktoren für $\mathrm{NaBa}_{3} \mathrm{RuO}_{6}$ (I) und $\mathrm{NaBa}_{3} \mathrm{IrO}_{6}$ (II) mit Standardabweichungen in Klammern.In der Raumgruppe $\mathrm{D}_{3 \mathrm{~d}}^{6}-\mathrm{R} \overline{3} \mathrm{c}$ sind folgende Punktlagen besetzt.

\begin{tabular}{lrccll}
\hline Atom & Lage & $x$ & $y$ & $z$ & $\mathrm{U}(\mathrm{eq})$ \\
\hline (I) & & & & & \\
$\mathrm{Na}$ & $6 \mathrm{a}$ & 0 & 0 & 0,2500 & $0,011(1)$ \\
$\mathrm{Ba}$ & $18 \mathrm{e}$ & $0,6432(1)$ & 0 & 0,2500 & $0,010(1)$ \\
$\mathrm{Ru}$ & $6 \mathrm{~b}$ & 0 & 0 & 0 & $0,007(1)$ \\
$\mathrm{O}$ & $36 \mathrm{f}$ & $0,1674(2)$ & $0,1499(2)$ & $0,0986(2)$ & $0,010(1)$ \\
$(\mathrm{II})$ & & & & & \\
$\mathrm{Na}$ & $6 \mathrm{a}$ & 0 & 0 & 0,2500 & $0,010(1)$ \\
$\mathrm{Ba}$ & $18 \mathrm{e}$ & $0,6439(1)$ & 0 & 0,2500 & $0,010(1)$ \\
$\mathrm{Ir}$ & $6 \mathrm{~b}$ & 0 & 0 & 0 & $0,007(1)$ \\
$\mathrm{O}$ & $36 \mathrm{f}$ & $0,1674(3)$ & $0,1501(3)$ & $0,0986(3)$ & $0,011(1)$ \\
\hline
\end{tabular}

\footnotetext{
* Weitere Einzelheiten zur Kristallstrukturuntersuchung können beim Fachinformationszentrum Karlsruhe, D-76344 Eggenstein-Leopoldshafen, unter Angabe der Hinterlegungsnummer (I) CSD 405133 und (II) CSD 405134 angefordert werden.
}

Tab. III. Interatomare Abstände $[\AA]$ für $\mathrm{NaBa}_{3} \mathrm{RuO}_{6}$ (I) und $\mathrm{NaBa}_{3} \mathrm{IrO}_{6}$ (II) mit Standardabweichungen in Klammern.
(I)

\begin{tabular}{|c|c|c|c|c|}
\hline $\mathrm{Na}-\mathrm{O}$ & $2,414(2)$ & $(6 x)$ & $\mathrm{Na}-\mathrm{O}$ & $2,420(3)$ \\
\hline $\mathrm{Ba}-\mathrm{O}$ & $\begin{array}{l}2,695(2) \\
2,717(2) \\
2,775(2) \\
2,878(2)\end{array}$ & $\begin{array}{l}(2 \mathrm{x}) \\
(2 \mathrm{x}) \\
(2 \mathrm{x}) \\
(2 \mathrm{x})\end{array}$ & $\mathrm{Ba}-\mathrm{O}$ & $\begin{array}{l}2,695(3) \\
2,725(3) \\
2,781(3) \\
2,889(3)\end{array}$ \\
\hline $\mathrm{Ru}-\mathrm{O}$ & $1,991(2)$ & $(6 x)$ & $\mathrm{Ir}-\mathrm{O}$ & $1,997(3)$ \\
\hline
\end{tabular}

Alle Rechnungen wurden auf einer IBM RS/ 6000 des Instituts für Anorganische Chemie der Universität Kiel durchgeführt.

\section{Diskussion}

Die Röntgenstrukturanalysen zeigen, daß $\mathrm{NaBa}_{3} \mathrm{RuO}_{6}$ und $\mathrm{NaBa}_{3} \mathrm{IrO}_{6}$ zum $_{\mathrm{Sr}_{4}} \mathrm{PtO}_{6}$-Typ gehören. Dieser wurde mehrfach ausführlich beschrieben $[3,4,10]$. Charakteristisch für diese Kristallstruktur sind längs [001] orientierte ${ }_{\infty}^{1}\left[\mathrm{NaRuO}_{6}\right]$-Polyederketten der Abfolge

$-\mathrm{NaO}_{6}($ Prisma $)-\mathrm{Ru} / \mathrm{IrO}_{6}($ Oktaeder $)$-. Die Verknüpfung zum dreidimensionalen Kristallverband erfolgt durch die Bariumionen. $\mathrm{Ba}^{2+}$ erhält mit den benachbarten $\mathrm{O}^{2-}$-Ionen der ${ }_{\infty}^{1}\left[\mathrm{NaRuO}_{6}\right]$-Polyederketten eine verzerrt quadratisch antiprismatische Umgebung. Der Vergleich mit dem Aristotyp $\mathrm{Sr}_{4} \mathrm{PtO}_{6}[10]$ zeigt, daß durch den Ersatz eines Erdalkalimetallions gegen $\mathrm{Na}^{+}$das Ladungsdefizit durch Anhebung der Oxidationsstufe des Edelmetalls von $\mathrm{M}^{4+}$ auf $\mathrm{M}^{5+}$ ausgeglichen wird. In der Phase $\mathrm{Sr}_{3.67} \mathrm{Na}_{0.33} \mathrm{PtO}_{6}$ [11] sollte somit Platin partiell fünfwertig sein, was jedoch nicht eindeutig bewiesen werden konnte. Bei Ersatz eines Erdalkalimetallions gegen $\mathrm{Cu}^{2+}$ bleibt in den Verbindungen $\mathrm{Sr}_{3} \mathrm{CuPtO}_{6}[12,13], \mathrm{Sr}_{3} \mathrm{CuIrO}_{6}[14]$ und $\mathrm{Ca}_{3} \mathrm{CuIrO}_{6}$ [15] die Oxidationsstufe des Edelmetalls zwar erhalten, Kupfer besetzt in den ${ }_{\propto}^{1}\left[\mathrm{NaRuO}_{6}\right]-$ Ketten jedoch nicht die Mitte der trigonalen $\mathrm{NaO}_{6}$-Prismen, sondern liegt nahe einer der rechteckigen Prismenflächen, wodurch eine quadratisch planare Koordination angestrebt wird. Eine Besonderheit weist $\mathrm{Ca}_{3.5} \mathrm{Cu}_{0.5} \mathrm{PtO}_{6}$ [16] auf. Hier liegt infolge Symmetrieerniedrigung eine geordnete Verteilung von $\mathrm{Ca}^{2+}$ und $\mathrm{Cu}^{2+}$ vor, so daß die $\mathrm{Ca}^{2+}$-Ionen eine trigonal prismatische und die $\mathrm{Cu}^{2+}$-Ionen eine fast planare Koordination erhalten. Neuerdings wurde Erdalkalimetall auch gegen $\mathrm{Zn}^{2+}$ und $\mathrm{Ni}^{2+}$ ersetzt [17]. Im Gegensatz zu Cu${ }^{2+}$ besetzt $\mathrm{Ni}^{2+}$ wie $\mathrm{Na}-$ trium in $\mathrm{NaBa}_{3} \mathrm{RuO}_{6}$ und $\mathrm{NaBa}_{3} \mathrm{IrO}_{6}$ die trigonalen Prismen. 
Dank

Der Deutschen Forschungsgemeinschaft und dem Fonds der chemischen Industrie danken wir für die Unterstützung mit wertvollen Sachmitteln.

[1] A. Tomaszewska, Dissertation Univ. Kiel (1993).

[2] G.Tams, Hk. Müller-Buschbaum, Z. Naturforsch. 49b, 585 (1994).

[3] S. Frenzen, Hk. Müller-Buschbaum, Z. Naturforsch. 50b, 581 (1995).

[4] S. Frenzen, Hk. Müller-Buschbaum, Z. Naturforsch. 51b, 225 (1996).

[5] S. Frenzen, Hk. Müller-Buschbaum, Z. Naturforsch. 51b, 485 (1996).

[6] P. D. Battle, S. H. Kim, A. V. Powell, J. Solid State Chem. 101, 161 (1992).

[7] S. A. Almaer, P. D. Battle, T. Lightfoot, R. S. Mellen, A. V. Powell, J. Solid State Chem. 102, 375 (1993).

[8] D. Y. Jung, G. Demazeau, J. Etourneau, M. A. Subramanian, Mater. Res. Bull. 30, 113 (1995).

[9] G.-M. Sheldrick. SHELXL-93, Program for Crystal Structure Determination, Göttingen (1993).
[10] J. J. Randall, L. Katz, Acta Crystallogr. 12, 519 (1959).

[11] G. Tams, Hk. Müller-Buschbaum, Z. Naturforsch. 49b, 581 (1994).

[12] A. P. Wilkinson, A. K. Cheetham, W. Kunnmann, A. Kvick, Eur. J. Solid State Inorg. Chem. 28, 453 (1991).

[13] J. L. Hodeau, H. Y. Tu, P. Bordet, T. Fournier, P. Strobel, M. Marezio, G. Chandrashekhar, Acta Crystallogr. B48, 1 (1992).

[14] M. Neubacher, Hk. Müller-Buschbaum, Z. Anorg. Allg. Chem. 607, 124 (1992).

[15] A. Tomaszewska, Hk. Müller-Buschbaum, Z. Anorg. Allg. Chem. 619, 534 (1993).

[16] A. Tomaszewska, Hk. Müller-Buschbaum, Z. Anorg. Allg. Chem. 617, 23 (1992).

[17] T. N. Nguyen, H. C. zur Loye, J. Solid State Chem. 117, 300 (1995). 